\title{
Pascal MARICHALAR, Qui a tué les verriers de Givors? Une enquête de sciences sociales
}

\section{Glenn Mainguy}

\section{(2) OpenEdition}

1 Journals

\section{Édition électronique}

URL : https://journals.openedition.org/ress/4713

DOI : $10.4000 /$ ress. 4713

ISBN : $1663-4446$

ISSN : 1663-4446

Éditeur

Librairie Droz

Édition imprimée

Date de publication : 30 mai 2019

Pagination : 283-286

ISSN : 0048-8046

\section{Référence électronique}

Glenn Mainguy, «Pascal MARICHALAR, Qui a tué les verriers de Givors? Une enquête de sciences sociales ", Revue européenne des sciences sociales [En ligne], 57-1 | 2019, mis en ligne le 05 février 2019, consulté le 04 janvier 2023. URL : http://journals.openedition.org/ress/4713 ; DOI : https://doi.org/ $10.4000 /$ ress. 4713

Ce document a été généré automatiquement le 4 janvier 2023.

Tous droits réservés 


\title{
Pascal MARICHALAR, Qui a tué les verriers de Givors? Une enquête de sciences sociales
}

\author{
Glenn Mainguy
}

\section{RÉFÉRENCE}

Pascal MARICHALAR, 2017, Qui a tué les verriers de Givors? Une enquête de sciences sociales, Paris, La Découverte, « L'envers des faits », p. 258

1 S'inscrivant dans le champ des études sur les maladies professionnelles, l'ouvrage de Pascal Marichalar propose une histoire de la mobilisation des anciens verriers de Givors, commune du département du Rhône, pour la reconnaissance de l'origine professionnelle des maladies qui les affligent. Se plaçant à l'extérieur du récit, l'auteur, en utilisant les outils de la sociologie et de l'histoire, retranscrit avec minutie, chapitre après chapitre, la chronologie de la mobilisation, démarrant par la découverte du cancer de Christian Cervantes en 2005 et se terminant par le procès des dirigeants de la verrerie aux prud'hommes en 2016. Au-delà des qualités rédactionnelle et narrative de l'auteur, l'originalité et la force de l'ouvrage tiennent aux choix effectués par celui-ci. Prenant délibérément le parti des verriers et choisissant comme point d'observation central le local de l'association des verriers, il propose les résultats d'une double enquête, à la fois celle menée par deux protagonistes centraux de l'ouvrage cherchant à prouver l'origine professionnelle des cancers, et la sienne qu'il qualifie lui-même d'« enquête sur l'enquête ». D'un point de vue méthodologique, le travail de Pascal Marichalar repose sur un important dispositif de recherche. Il a suivi pendant trois ans, de 2013 à 2016, les activités de l'association des anciens verriers de Givors et il a effectué une cinquantaine d'entretiens avec les ouvriers. Il a eu accès à une somme importante d'archives personnelles et il a analysé les articles de presse et les décisions de justice qui concernent les affaires de maladies professionnelles. Rendre compte de la 
richesse de cet ouvrage aux allures de chronique judiciaire ne peut se faire qu'en retraçant, avec l'auteur, le cheminement de la mobilisation.

Pascal Marichalar ouvre son récit (chapitre premier) en retraçant l'histoire de la verrerie de sa naissance en 1749 à sa fermeture en 2003. Il revient plus particulièrement sur la très "belle opération " financière $d^{\prime}$ '" achat avec effet de levier » qui a conduit au rachat de l'usine par la société Owens-Illinois Manufacturing et à la « dernière coulée ». L'auteur relate, ensuite, les différentes étapes du combat des verriers contre la fermeture. Malgré la lutte syndicale, le plan social est adopté. Les revendications des ouvriers se transforment alors pour se focaliser sur les conditions de la fermeture. C'est au cours de cette période qu'ils demandent à conserver un local au sein de l'usine afin qu'une Association des anciens verriers de Givors y établisse son siège ; association qui va devenir, après la découverte du premier cancer de Christian Cervantes, le point central de la mobilisation des verriers pour la reconnaissance de l'origine professionnelle de leurs maladies.

Le processus faisant émerger la problématique des maladies professionnelles apparait au second chapitre, à travers la description des premières étapes de la mobilisation. Pascal Marichalar y expose le travail d'enquête réalisé par Mercedes Cervantes, épouse de Christian, et Laurent Godon, imprimeur, historien et «intellectuel public» (p. 47), pour mettre au jour la possible origine professionnelle des cancers. Ensemble, ils réalisent une vaste "épidémiologie populaire » qui révèlera l'existence d'une surreprésentation des cancers chez les anciens verriers et de ce que l'auteur qualifie de "scène de crime". À travers la retranscription des paroles des verriers, l'auteur montre la difficile reconnaissance par ces derniers du rôle de la verrerie dans la survenue des cancers et la pénible acceptation par ceux-ci de la mise en cause de leur ancien employeur : «tu ne mords pas la main qui t'a nourri » (p. 54). Il rappelle ici la force symbolique du cadre paternaliste qui structurait la relation entre les verriers et les dirigeants de l'usine.

Contre la mise en scène, par les industriels, de la pureté du verre et de l'innocuité du processus de fabrication, Pascal Marichalar revient, dans le troisième chapitre, sur l'histoire de l'industrie verrière à travers la description des conditions de travail existantes au sein de ces "abatteuses d'hommes" (p.64) où les verriers sont de «la viande à feu » (p.68). Après un détour par l'histoire industrielle, l'auteur reprend le récit du travail de Laurent Gonon. Celui-ci a fait appel à la mémoire des ouvriers afin qu'ils lui exposent les conditions d'exercice de leur travail. Grâce aux souvenirs recueillis, il a pu identifier, d'un côté les produits chimiques entrant dans le processus de fabrication du verre, et d'un autre côté les modes d'exposition des verriers à ces produits. Par ce biais, il dresse un «liste impressionnante de causes reconnues de maladie auxquelles les ouvriers étaient exposés [...] sans qu'aient été prises les mesures de prévention nécessaire» (p. 91). C'est après la présentation des résultats de cette enquête que les ouvriers entameront une action dans le but d'obtenir "une reconnaissance collective de l'état de maladie professionnelle » (p. 92).

5 Le quatrième chapitre analyse le moment où « l'action de l'association se mue [...] en combat pour que justice soit rendue» (p.93). Constituant la famille Cervantes en véritable cas, Pascal Marichalar y rend compte de l'engagement des verriers dans une procédure de reconnaissance de maladie professionnelle auprès de l'assurance-maladie " selon un protocole qui n'a que très peu changé depuis la première loi sur les maladies professionnelles en $1919 »$ (p.94). Si l'origine professionnelle d'une maladie n'est pas 
admise par la loi, comme dans le cas des verriers de Givors, le travailleur « doit luimême apporter les preuves qu'il y a un lien "direct et essentiel" entre son activité professionnelle et sa maladie » (p.115). À travers le récit de cette procédure, l'auteur témoigne de la confrontation des ouvriers à la bureaucratie administrative. Il interroge les rapports entre le monde ouvrier et le monde médical. Il fait apparaître la domination symbolique qui s'exerce sur les verriers. Se concentrant sur les services de la médecine du travail, il rapporte les difficultés rencontrées par les ouvriers pour obtenir leur dossier médical, ainsi que les procédures d'expurgation menées par ces services pour limiter la divulgation d'information sur leur état de santé. Une conclusion s'impose alors à l'auteur à propos de ce "gouvernement des travailleurs malades ": il s'agit de « restreindre le nombre de personnes qui seront reconnues, tout en projetant l'apparence d'une procédure qui se fonderait uniquement sur la connaissance scientifique » (p. 94).

6 L'auteur relate, dans le cinquième chapitre, l'arrivée de nouveaux acteurs - celle d'une infirmière «militante syndicale » (p. 117) ainsi que celle des membres du "Giscop 93 » - dans la mobilisation des verriers. Leur volonté, écrit Pascal Marichalar, est d'aider les ouvriers à produire la preuve de leur exposition en réalisant une " contre-expertise » fondée sur la technique du curriculum laboris. À travers la description de cette collaboration, l'auteur nous donne à lire « une rencontre entre deux mondes que rien ne prédisposait à se fréquenter, celui des militants ouvriers et celui des professionnels de la science » (p. 122). La seconde partie du chapitre est consacrée à la description des procédures de jugement des deux cancers de Christian Cervantes devant le tribunal des affaires de sécurité sociale. Le récit de cette action en justice est l'occasion pour l'auteur d'évoquer l'entrée en scène aux côtés des verriers du cabinet Tessonnière « ultraspécialisé sur la question des maladies professionnelles» (p. 129). Étayé par les recherches de scientifiques « engagés », le travail des avocats sera de démontrer devant le tribunal l'existence d'une "relation causale essentielle et directe» (p. 142) entre la maladie de Christian Cervantes et son activité professionnelle au sein de la verrerie. M. Cervantes obtiendra définitivement la reconnaissance de l'origine professionnelle de ces deux cancers en novembre 2015, c'est-à-dire presque quatre ans après son décès.

7 À partir du sixième chapitre, l'auteur met au jour une nouvelle transformation dans la stratégie des verriers. Il ne s'agit plus seulement pour eux de faire en sorte que l'origine professionnelle de leur maladie soit reconnue, mais aussi que les responsables, ces "meurtriers sans visage », soient condamnés. Pascal Marichalar place au cœur de ce chapitre la question du caractère criminel ou non de l'exposition de travailleurs par des responsables d'entreprises à un risque sanitaire connu. Son propos s'organise autour de l'instruction menée par le parquet de Lyon après la plainte déposée par Christian Cervantes. L'auteur y mentionne les auditions des témoins, l'expertise médicale réalisée afin de décrire l'état de santé de Christian. Finalement, le juge prononcera un non-lieu justifié par l'impossibilité de démontrer le lien causal entre la survenue du cancer et le travail de M. Cervantes dans la verrerie. À partir du cas de Christian, ce chapitre propose une réflexion critique sur le code pénal et sur son inadaptation aux affaires de maladies industrielles.

8 Le septième chapitre vient clore le rapport de l'affaire des verriers à travers la description du procès qui s'est tenu au tribunal des prud'hommes le 2 septembre 2014 en mettant face à face les verriers et la société Owen-Illinois Manufacturing. L'auteur y décrit avec précision le déroulement de ce "procès de la peur" en exposant les 
différentes plaidoiries des avocats. Celui des verriers accuse les responsables de la verrerie d'avoir exposé les ouvriers à des produits dangereux pour leur santé (amiante, CMR, etc.) et demande une indemnisation au titre du préjudice causé par l'anxiété liée à cette exposition. Celle de la partie adverse appelle à ne pas faire de "doux mélanges » (p. 174) entre l'amiante et les CMR et défend l'innocuité des produits chimiques utilisés dans le processus de fabrication du verre "allant même jusqu'à les qualifier d'“huiles propres" » (p. 175). Après avoir rappelé l'histoire de la procédure de reconnaissance du préjudice d'anxiété, l'auteur reprend le récit du procès. Malgré la promesse du juge de dégager une majorité, l'affaire des verriers est renvoyée au départage et sera rejugée en février 2016. Les deux visions de l'affaire incarnées par la voix des avocats s'affrontent de nouveau. «La décision est rendue trois mois plus tard, le 28 avril 2016. Elle est négative pour les verriers » (p. 190).

Dans le huitième et dernier chapitre, nous quittons le monde des verriers de Givors. Pascal Marichalar y évoque ses visites au sein d'autres verreries françaises. Il cherche à savoir comment ont évolué les conditions de travail des ouvriers depuis la fermeture de la verrerie de Givors en 2003. Malgré quelques aménagements apportés aux postes de travail, il montre que « les dangers identifiés par Mercedes Cervantes et Lauren Gonon dans l'usine de Givors étaient encore bien présents dans les verreries industrielles françaises » (p. 198). Pascal Marichalar montre, ensuite, comment les nouvelles réglementations environnementales participent à la production de nouveaux métiers à risques. Enfin, il termine sa description du « cycle du sacrifice » en exposant comment les nouvelles techniques de management poussent une partie toujours plus précarisée du monde ouvrier à accepter de faire des tâches toujours plus dangereuses pour leur santé.

10 En somme, l'ouvrage de Pascal Marichalar est un exemple réussi d'apport à l'analyse et à la compréhension des problèmes sociaux par le croisement des approches sociologique et historique. Plus qu'un travail portant sur la question des maladies professionnelles, cet ouvrage propose une réflexion sur la sociologie de la justice en faisant apparaître la difficulté de penser le juste : «s'agit-il d'obtenir des sanctions, des compensations, l'assurance que cela ne se reproduira plus, des excuses, des expressions d'empathie? » (p. 93). À travers une analyse fine des rapports sociaux, il montre que dans le cas d'un groupe social subalterne, la difficile réparation d'une injustice tient autant aux mécanismes sociaux de prise en charge de cette injustice qu'à la difficulté pour les individus de savoir précisément comment doit être réparée l'injustice qui a été commise.

11 Toutefois, la force de l'ouvrage de Pascal Marichalar est aussi sa faiblesse: la focalisation sur le groupe social des verriers permet à l'auteur de rendre compte avec exactitude du combat des verriers et de leur rapport « dominé » aux institutions, mais sa tendance à réifier le collectif des verriers et à le constituer comme un groupe social uni, laisse dans l'ombre la description de leurs relations interindividuelles et les modalités de leur engagement individuel dans la lutte. Le lecteur aurait été heureux de connaître également les débats, les discordances internes et les conflits qui structurent tout collectif d'individus.

12 Pour finir, l'auteur nous offre ici un remarquable livre sur la condition salariale contemporaine, condition marquée par la permanence du tiraillement «entre la défense de l'emploi et la préservation de la santé » (p. 13). 


\section{AUTEURS}

\section{GLENN MAINGUY}

Sciences Po Bordeaux - Centre Émile Durkheim 